\title{
Neue Medikamente in der Onkologie: Nutzen, Risiken und Herausforderungen
}

\author{
David Cella ${ }^{a}$ Volker Vervölgyi ${ }^{b} \quad$ Bernhard Wörmann ${ }^{c}$ \\ ${ }^{a}$ Department of Medical Social Sciences, Northwestern University, Chicago, IL, USA \\ ${ }^{b}$ Institut für Qualität und Wirtschaftlichkeit im Gesundheitswesen (IOWiG), Köln, \\ ${ }^{c}$ Deutsche Gesellschaft für Hämatologie und Medizinische Onkologie (DGHO), Berlin, Deutschland
}

\section{Der Nutzen für den Patienten: Zur Erfassung der gesundheitsbezogenen Lebensqualität}

\section{Verantwortlicher Autor: David Cella, Chicago}

Die Frage, anhand welcher Erfolgsparameter (Studienendpunkte) sich der Nutzen neuer onkologischer Therapien im Rahmen von Studien am besten messen lässt, wird derzeit allerorten kontrovers diskutiert. Goldstandard für den Nachweis eines klinischen Nutzens ist die Messung der Gesamtüberlebenszeit (overall survival (OS)). Dieser Endpunkt wird zunehmend ergänzt durch weitere Parameter wie das progressionsfreie Überleben (PFS). Beide Endpunkte sind von hoher Relevanz, reflektieren jedoch nicht unmittelbar den Patientennutzen. Subjektive, vom Patienten zu beschreibende Veränderungen wie eine Symptomlinderung oder die Steigerung der körperlichen Leistungsfähigkeit sollten deshalb häufiger als bisher als zusätzliche Studienendpunkte gewählt werden [1-4]. Fragebögen zur Erfassung patientenrelevanter Outcome-Parameter (patient-reported outcomes, PROs) bieten viele Vorteile: So erlauben sie unter anderem die Beurteilung von Therapieeffekten und der Schwere der Symptomatik aus Sicht der Patienten, wobei die formale Erfassung mittels Fragebögen die Auswertung der Angaben erleichtert.

Medizinische Interventionen können sich auf die körperliche, mentale und soziale Gesundheit eines Patienten auswirken. Insofern wird mit der Erfassung der PROs ein wesentlicher Beitrag zur Einschätzung des Therapieerfolges geleistet. Dies gilt in besonderem Maße für onkologische Therapiestudien, da Veränderungen in den PROs bzw. in der gesundheitsbezogenen Lebensqualität (health-related quality of life (HRQOL)) mit objektiv zu erfassenden Parametern wie Tumoransprechen und Überlebenszeit korrelieren. Auch kann eine symptomatische Verschlechterung ein früher Hinweis auf eine Progression der Erkrankung sein $[3,5]$. In einer Metaanalyse von 21 Studien konnte gezeigt werden, dass radiologisch erfasste Tumormerkmale (Remission, stabile Erkrankung, Progression) mit subjektiven Veränderungen der Beschwerdelast assoziiert sind [6], wobei die Effektstärke allerdings relativ gering ist.

Für die Erfassung der Lebensqualität während onkologischer Therapien steht mittlerweile eine Reihe von multidimensionalen Lebensqualitätsfragebögen zur Verfügung, die sowohl allgemeine als auch krankheitsspezifische Aspekte der Lebensqualität abbilden. So werden zum Bespiel für Therapiestudien beim fortgeschrittenen Nierenzellkarzinom die (allgemeinen) Fragebögen SF 36, EQ-5D, HUI II/III und RSCL sowie die (krankheitsspezifischen) Fragebögen FACTG (plus Subskalen), FACT Symptom Indexes und EORTCQLQ (plus Zusatzmodule) angewendet [7].

\section{Lebensqualität frühzeitig miterfassen}

Mit der Erhebung der Lebensqualität im Rahmen klinischer Studien sollte bereits in Phase II der klinischen Arzneimittelentwicklung und somit weit vor der avisierten Zulassung begonnen werden. Dies erfordert eine sorgfältige Wahl der PRO-Instrumente im Hinblick auf die Zielsetzung der geplanten Studien: Geht es hauptsächlich um den Nachweis der überlegenen Wirksamkeit der neuen Substanz, um eine Verringerung der Krankheitslast oder um individuelle Therapiepräferenzen? Bei entsprechender strategischer Planung ermöglichen bereits Phase-II-Studien die Abschätzung der Effektivität und eine Erfassung der therapiebedingten Toxizität. Die gewählten Studienendpunkte sowie die Instrumente zu deren Erfassung sollten bereits frühzeitig mit den Zulassungsbehörden abgestimmt werden.

Die heute zur Verfügung stehenden Standardinstrumente zur Lebensqualitätserfassung in der Onkologie haben alle

\begin{tabular}{ll}
\hline KARGER & (1) 2013 S. Karger GmbH, Freiburg \\
$0378-584 X / 13 / 3617-0012 \$ 38.00 / 0$ \\
Fax +49 761 4520714 \\
$\begin{array}{l}\text { Information@Karger.com } \\
\text { www.karger.com }\end{array}$ & $\begin{array}{l}\text { Accessible online at: } \\
\text { www.karger.com/onk }\end{array}$
\end{tabular}


Abb. 1. Lebensqualität (mit dem TOI-Score erfasst) als Prädiktor (modifiziert nach [10]).

\section{Gesamt- überleben}

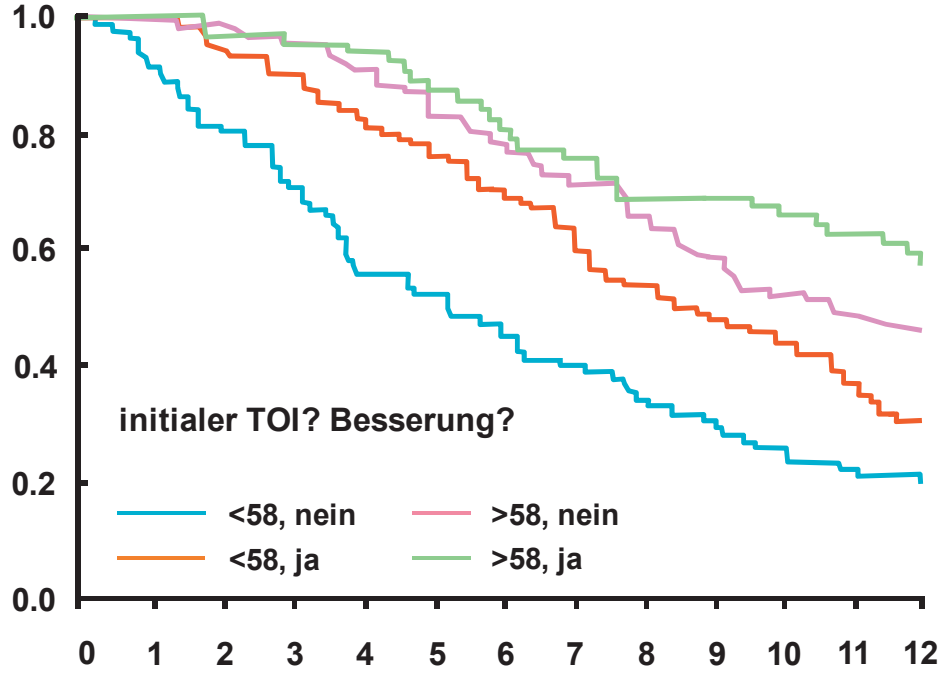

Tab. 1. PROs: Unterschiede zwischen Verum (Erlotinib) und Placebo (modifiziert nach [11])

\begin{tabular}{|c|c|c|c|c|c|}
\hline & \multicolumn{2}{|l|}{ Erlotinib } & \multicolumn{2}{|l|}{ Placebo } & \multirow{2}{*}{$\begin{array}{l}\text { Hochberg } \\
\text { adjusted } \\
\text { p value }\end{array}$} \\
\hline & Patienten, $\mathrm{n}$ & $\begin{array}{l}\text { Median (95\%- } \\
\text { CI), Monate }\end{array}$ & Patienten, $\mathrm{n}$ & $\begin{array}{l}\text { Median ( } 95 \% \text { - } \\
\text { CI), Monate }\end{array}$ & \\
\hline Husten & 298 & $4,9(3,8-7,4)$ & 153 & $3,7(2,0-4,9))$ & 0,04 \\
\hline Dyspnoe & 253 & $4,7(3,8-6,2)$ & 179 & $2,9(2,0-4,8)$ & 0,03 \\
\hline Schmerz & 348 & $2,8(2,4-3,0)$ & 179 & $1,9(1,8-2,8)$ & 0,04 \\
\hline
\end{tabular}

$\mathrm{CI}=$ Konfidenzintervall einen umfangreichen Validierungsprozess einschließlich der Überprüfung ihrer psychometrischen Eigenschaften durchlaufen. Sie bestehen aus einem Kernfragebogen, der für die meisten Tumorerkrankungen anwendbar sein sollte. Er enthält Fragenkomplexe zu den 3 wichtigen Kernbereichen der Lebensqualität: dem körperlichen Bereich, dem psychischseelischen Bereich sowie dem sozialen Bereich. Des Weiteren finden sich im Kernfragebogen Fragen zu häufig auftretenden Symptomen und allgemeinen Beschwerden. Ergänzt werden die Kernfragebögen durch Ergänzungsbögen, sogenannte Module, die die für spezielle Situationen notwendigen Zusatzfragen z.B. hinsichtlich Symptomatik oder Nebenwirkungen der Behandlung enthalten. Viele der Standardfragebögen stehen in mehreren Sprachen zur Verfügung.

\section{FACT und EORTC-QLQ sind häufig verwendete \\ Messinstrumente}

In Nordamerika hat sich der FACT-Fragebogen [8] als Standardinstrument etabliert. Er verfügt im Vergleich zu anderen Fragebögen zur Lebensqualität über die größte Sammlung spezifischer Module. So besteht etwa die in 49 Sprachen (inklusive Deutsch) übersetzte Subskala FACT-L (Functional Assessment of Cancer Therapy-Lung-Skala) insgesamt aus etwa 40 Fragestellungen, die von den Patienten selbst anhand einer 5-stufigen Likert-Skala (0-4) zu beantworten sind und
Teilaspekte der Lebensqualität der vergangenen Woche betreffen. Die Fragen verteilen sich auf 6 Subskalen: körperliches Wohlbefinden (PWB), soziales/familiäres Wohlbefinden (SWB), das Verhältnis zum behandelnden Arzt, emotionales Wohlbefinden (EWB), funktionales Wohlbefinden (FWB) und die Lungenkrebs-Subskala (LCS), die mit lediglich 7 Fragen präzise auf die Lungenkrebs-assoziierte Symptomatik fokussiert. Die Auswertung des Bogens erfolgt anhand eines Punktesystems, sowohl für die einzelnen Subskalen als auch summarisch. Ein höherer Punktewert entspricht einer höheren Lebensqualität. Die Summe der PWB-, FWB- und LCSSkalen ist das Maß der physischen Lebensqualität und wird als FACT-L TOI (trial outcome index) bezeichnet. Der FACT-L TOI wird insbesondere im Rahmen von klinischen onkologischen Studien eingesetzt, da seine Ergebnisse als präzise Lungenkrebs-spezifische Lebensqualitätsindikatoren gelten, während der gesamte FACT-L auch für andere Lungenerkrankungen nutzbar ist. Mithilfe der FACT-LCS-Skala und des FACT-L TOI lassen sich recht zuverlässig Unterschiede in der Lebensqualität in Abhängigkeit von verschiedenen klinischen Indikatoren beschreiben [9]. Als klinisch relevante Verbesserung gilt eine Zunahme von mindestens 6 Punkten im FACT-L oder TOI bzw. von mindestens 2 Punkten im FACT-LCS innerhalb von 4 Wochen. In einer PhaseIII-Studie mit Lungenkrebspatienten (Eastern Cooperative 
Oncology Group (ECOG)-Studie 5592) erwiesen sich sowohl der TOI-Scorewert zu Studienbeginn als auch die Veränderung des TOI-Scores im Therapieverlauf als Prädiktoren für das Tumoransprechen und korrelierten mit der Überlebenszeit [10] (Abb. 1).

Auch mit dem vornehmlich in Europa verwendeten Fragebogen EORTC-QLQ (European Organisation for Research and Treatment of Cancer) lassen sich die Lebensqualität und deren Veränderung im Verlauf der Therapie zuverlässig erfassen. Dies hat sich unter anderem in einer placebokontrollierten Studie zur Behandlung von LungenkrebsPatienten mit Erlotinib gezeigt [11]. Die Zeit bis zur Verschlechterung verschiedener PROs (Husten, Dyspnoe, Schmerzen) war in der Verumgruppe signifikant länger als in der Placebogruppe (Tab. 1). Da sich anhand solcher Parameter sicher zwischen Behandlungseffekten diskriminieren lasst, dürften sie als Endpunkte in klinischen Studien weiter an Bedeutung gewinnen.

Um die krankheitsbezogene Lebensqualität möglichst genau abzubilden, wurden für zahlreiche Tumorentitäten spezielle Indizes entwickelt. Diese Erhebungsbögen können für anstehende Therapieentscheidungen hilfreich sein oder als zusätzliche Information in Leitlinien integriert werden. Der FACT-Kidney Symptom-Index (FKSI) enthält in der Langform 15 Fragen [12] und wurde unter anderem als sekundärer Endpunkt in einer Therapiestudie (Axitinib vs. Sorafenib; AXIS-Studie) bei Nierenkrebs verwendet [13].

Um PROs übergreifend bei einem möglichst großen Spektrum von Erkrankungen zuverlässig erfassen zu können, haben die amerikanischen National Institutes of Health (NIH) das Patient-Reported Outcomes Measurement Information System (PROMIS ${ }^{\circledR}$ ) konzipiert [14]. Es verfolgt das Ziel, die vorhandenen Fragebogen-basierten Datenbanken weiterzuentwickeln und $\mathrm{zu}$ validieren, um sie generisch bei verschiedenen Erkrankungen einsetzen zu können. Ein Vorteil der Verwendung international verbreiteter Fragebögen besteht unter anderem in der besseren Vergleichbarkeit national erhobener Daten. Einige PROMIS-Datenbanken (Depressivität, Angsterkrankungen) wurden bereits ins Deutsche übersetzt.

\section{Herausforderungen aus Sicht des Onkologen}

\section{Verantwortlicher Autor: Bernhard Wörmann, Berlin}

Die Onkologie steht heute vor großen Herausforderungen, da sie für eine stetig wachsende Zahl von Patienten die jeweils wirksamsten, den Kriterien der evidenzbasierten Medizin genügenden Therapien bereitstellen soll. Die aktuelle Entwicklung geht in Richtung einer stärker personalisierten Medizin und erfordert eine entsprechende Nutzen-RisikoAbwägung für jeden einzelnen Patienten. Im Spannungsfeld zwischen Innovation und Kostenexplosion soll der Onkologe bei der Therapiefindung einen Mittelweg finden, der auch die Erwartungen des Patienten an eine Krebstherapie einschließlich der ethisch-emotionalen Aspekte berücksichtigt. Krebs ist vielleicht nicht der «König aller Krankheiten» [15], aber eine seit Jahrtausenden bestehende Herausforderung.

\section{Die demografische Entwicklung als Herausforderung}

Die Bevölkerung in Deutschland wird sich ausgehend vom Jahr 2008 innerhalb von 12 Jahren um etwa 2,2\% verringern, das entspricht einem Bevölkerungsverlust von etwa 1,8 Millionen Menschen (nach Berechnungen des Instituts für Community Medicine, Universität Greifswald). Der Bevölkerungsrückgang wird voraussichtlich regional sehr unterschiedlich ausfallen und in einigen nördlichen und östlichen Regionen mehr als $10 \%$ betragen. Im gleichen Zeitraum wird die Zahl der neu diagnostizierten Krebserkrankungen deutlich zunehmen, und zwar um 9\% (+21 000 Erkrankungen pro Jahr) bei Frauen und um 18\% (+46 000 Erkrankungen pro Jahr) bei Männern. Dementsprechend wird die Krebsprävalenz um 60000 (Frauen) bzw. 116000 (Männer) steigen.

\section{Die personalisierte Medizin stellt neue Aufgaben}

Die Chancen und Herausforderungen im Zusammenhang mit einer stärker personalisierten Medizin lassen sich anschaulich am Beispiel des nichtkleinzelligen Lungenkarzinoms (NSCLC) verdeutlichen. Bis vor wenigen Jahren gab es für Patienten in fortgeschrittenen Krankheitsstadien nur wenige Behandlungsmöglichkeiten. Mithilfe moderner Labormethoden sind mittlerweile mehr als 1000 genetische Merkmale (z.B. EGFR-aktivierende Mutationen, ALK-Translokationen (ALK = Anaplastic-Lymphoma-Kinase, EGFR = epidermaler Wachstumsfaktorrezeptor)) beim NSCLC detektiert worden, von denen einige bereits als therapeutisches Target genutzt werden. Das Therapieangebot hat sich mit diesen zielgerichteten Therapien deutlich erweitert, allerdings müssen die Behandlungskonzepte für jeden Patienten anhand der histologischen und molekulargenetischen Tumormerkmale individuell ausgearbeitet werden.

\section{Zielgerichtete Therapien haben Eingang in die Leitlinien gefunden}

Über viele Jahre wurde die Chemotherapie bei Patienten mit NSCLC ausschließlich nach klinischen Kriterien wie Komorbidität und Allgemeinbefinden ausgewählt. Heute sollten bei nicht operablen Tumoren grundsätzlich die Ergebnisse genetischer Untersuchungen mit herangezogen werden, bevor mit einer systemischen Therapie begonnen wird. Auch für Patienten mit weit fortgeschrittener Erkrankung können in Abhängigkeit vom ECOG-Status und den Tumormerkmalen verschiedene Therapien eingesetzt werden, die zu einer Verlängerung der progressionsfreien Zeit führen können. Die Leitlinien der Deutschen Gesellschaft für Hämatologie und Medizinische Onkologie (DGHO) [16] empfehlen die Erhebung des EGFR-Mutationsstatus und des ALK-Translo- 


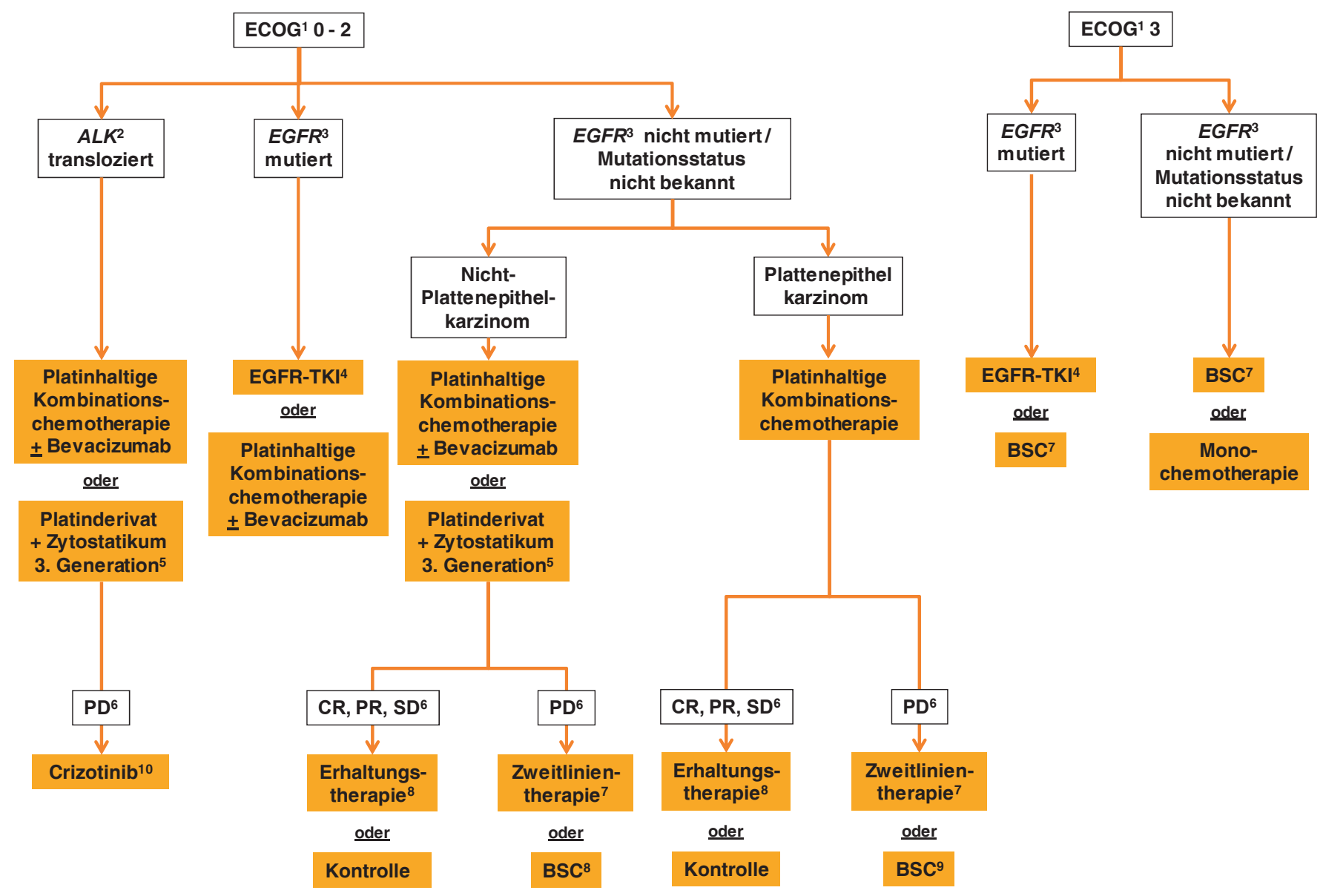

Abb. 2. NSCLC Stadium IV: Therapiealgorithmus für die palliative medikamentöse Erstlinientherapie [16]. (1) ECOG - Klassifikation des Allgemeinzustandes; (2) ALK - Gen der Anaplastic-Lymphoma-Kinase; (3) EGFR - Gen des epidermalen Wachstumsfaktorrezeptors; (4) EGFR-TKI - EGFR-Tyrosinkinaseinhibitoren; (5) Zytostatika 3. Generation - Gemcitabin, Taxane, Vinorelbin; (6) CR - komplette Remission, PR - partielle Remission, SD - stabile Erkrankung; (7) Erhaltungstherapie - Bevacizumab bei Nicht-Plattenepithelkarzinom und nach Vorbehandlung mit Bevacizumab; Erlotinib unabhängig von Histologie und Vorbehandlung; Pemetrexed bei Adenokarzinom und nach Vorbehandlung mit Pemetrexed; (8) Zweitlinientherapie siehe [16] Kapitel 5.1.12.1; (9) BSC - Best Supportive Care; (10) beachte Zulassungsstatus.

kationsstatus aller Patienten mit nichtplattenepithelialem NSCLC, unabhängig vom Raucherstatus vor Beginn der medikamentösen Erstlinientherapie. Positive Studienergebnisse zum Einsatz der neuen Substanzen führten zu entsprechenden Therapieempfehlungen (Abb. 2) unter anderem für die Anwendung der oralen Tyrosinkinaseinhibitoren Erlotinib und Gefitinib bei aktivierender EGFR-Mutation. Damit behandelte Patienten erreichen höhere Tumorremissionsraten und ein längeres progressionsfreies Überleben im Vergleich zur konventionellen Chemotherapie. Bei Patienten mit dem Tumormerkmal einer ALK-Mutation führt die Zweitlinientherapie mit dem Tyrosinkinaseinhibitor Crizotinib zu einer signifikanten Verlängerung des progressionsfreien Überlebens.

Die Aktualisierung klinischer Leitlinien erfolgt auf der Grundlage evidenzbasierter Studien, die in der Regel als randomisierte, kontrollierte Phase-III-Studien konzipiert sind. Die für solche Studien erforderliche große Zahl an Patienten ist umso schwerer zu erreichen, je seltener die jeweilige Er- krankung ist. In Deutschland ist die pädiatrische Onkologie bei der Einbringung von Patienten in Studien ein Vorbild. Etwa 90\% der an Krebs erkrankten Kinder und Jugendlichen wurden bisher im Rahmen klinischer Studien behandelt. Bei Erwachsenen gelingt das nicht, da ein beträchtlicher Teil der Patienten z.B. wegen Komorbidität nicht die Vorraussetzungen für eine Studienteilnahme erfüllt. Bei den zahlenmäßig selteneren, hämatologischen Tumoren nehmen bis zur Hälfte der Patienten an Therapiestudien teil, bei den häufig auftretenden soliden Tumoren wie Darm- und Lungenkrebs sind es jedoch weniger als $10 \%$. An den zulassungsrelevanten Studien für zielgerichtete Therapien nehmen noch weniger Patienten teil, da nur jeweils ein Teil von ihnen die zusätzlich geforderten genetischen Tumormerkmale aufweist. Solche Studien werden häufig weltweit durchgeführt, um in vertretbarer Zeit genügend Teilnehmer in die Studie aufnehmen zu können. Daraus ergibt sich allerdings das Problem, das entweder große Gruppen von Patienten mit ganz unterschiedlichem ethnischen Hintergrund gebildet werden müssen oder meh- 


\section{RECIST-Kriterien}

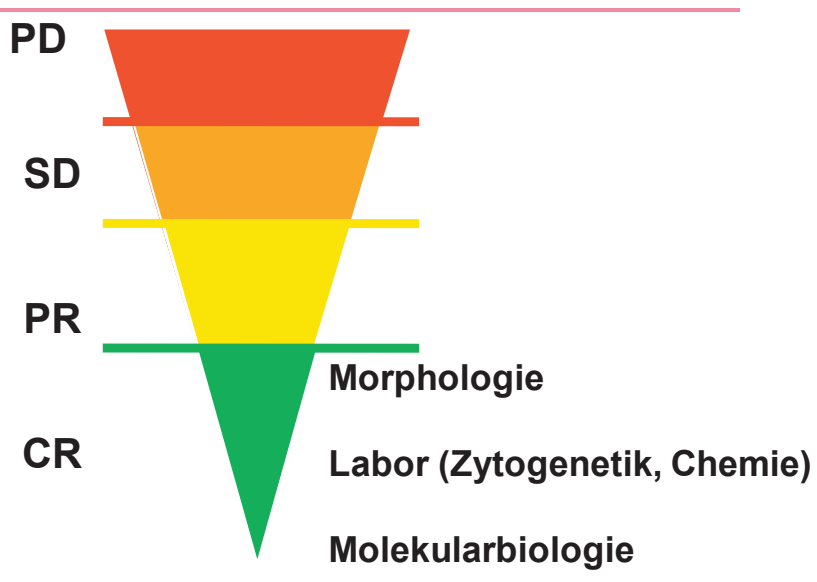

Abb. 3. RECIST; in Anlehnung an [17].

rere kleinere Studien aus verschiedenen Kontinenten für die Datenanalyse zusammengeführt werden. Je nach der genetischen Ausstattung (genetischer Polymorphismus) eines Menschen werden Medikamente jedoch unterschiedlich metabolisiert, sodass unter Umständen bei asiatischen Patienten ein Medikament anders wirkt als bei europäischen Patienten. Vor diesem Hintergrund muss sichergestellt werden, dass bei der Nutzen-Risiko-Abschätzung neuer Wirkstoffe genügend Studiendaten mit regionalem Bezug vorliegen. Außerdem ist zu beachten, dass die am häufigsten in klinischen Studien gewählten Endpunkte - das Gesamtüberleben und die Lebensqualität - einer Reihe von Einschränkungen hinsichtlich der Nutzen-Risiko-Bewertung unterliegen. So wird das Gesamtüberleben von Patienten nicht nur durch die Effektivität der Prüfsubstanzen beeinflusst, sondern durch zahlreiche weitere Faktoren im Verlauf der Krankheit. Das gilt insbesondere für Erstlinientherapie-Studien, bei denen das Gesamtleben der Patienten auch durch nachfolgende Zweit- und Drittlinientherapien bestimmt wird. Die Messung der Lebensqualität kommt insbesondere in der palliativen Situation den tatsächlichen Patientenbedürfnissen oft näher. Allerdings ist auch die Lebensqualität ein schwer zu objektivierender Parameter, wie dem Beitrag von D. Cella zu entnehmen ist.

\section{Progresssionsfreies Überleben als Studienendpunkt}

Als weitere Studienendpunkte werden häufig Parameter des Tumoransprechens gewählt, wobei sich für solide Tumoren die Einteilung nach RECIST (Response Evaluation Criteria in Solid Tumors) bewährt hat [17] (Abb. 3). Zunehmend wird auch in Phase-III-Studien das progressionsfreie Überleben als primärer Endpunkt gesetzt, etwa wenn wie bei der Erstlinientherapie des kolorektalen Karzinoms mehrere weitere Therapielinien verfügbar sind, die den Überlebensvorteil durch ein Erstlinienregime verzerren könnten.
Für hämatoonkologische Tumoren wie etwa Leukämien und Lymphome gelten die RECIST nicht, da hier inzwischen zunehmend auch biochemische oder molekulargenetische Parameter zur Bestimmung der Remissionsqualität und für das Monitoring angewendet werden.

\section{Das Instrument der Frühen Nutzenbewertung}

Die Diskussion über den Stellenwert patientenbezogener Endpunkte wird in Deutschland auch vor dem Hintergrund der gesetzlich vorgegebenen sogenannten Frühen Nutzenbewertung geführt. Mit Inkrafttreten des Arzneimittelmarktneuordnungsgesetzes (AMNOG) Anfang 2011 werden neue Medikamente nach der arzneimittelrechtlichen Zulassung hinsichtlich ihres Zusatznutzens gegenüber bereits verfügbaren Therapien bewertet. Abhängig vom Ergebnis dieses Verfahrens wird für das Präparat ein Rabatt zu dem initial frei vom pharmazeutischen Unternehmen festgelegten Preis verhandelt. Die Höhe des Rabattes ist abhängig vom durch den Gemeinsamen Bundesausschuss (G-BA) festgestellten $\mathrm{Zu}$ satznutzen des neuen Arzneimittels.

Formal werden bei der Ermittlung des Zusatznutzens eines Präparates bei Hinzuziehung des Instituts für Qualität und Wirtschaftlichkeit im Gesundheitswesen (IQWiG) nicht nur die Mortalität, sondern auch Morbidität, Lebensqualität und Nebenwirkungen berücksichtigt. Die Realität sieht oft anders aus: Seit Anfang 2012 haben 10 onkologische Präparate die Frühe Nutzenbewertung durchlaufen. Bei der Hälfte hat sich die Bewertung primär auf Daten zum Gesamtüberleben gestützt, erst in letzter Zeit wurden auch weitere Endpunkte wie das progressionsfreie Überleben stärker gewichtet. Der Grad des Zusatznutzens (erheblich, beträchtlich oder gering) ist letztlich vom G-BA festzulegen.

In mehreren Fällen fielen die Einschätzungen des IQWiG, der hämatologisch-onkologischen Experten und des G-BA unterschiedlich aus, ungeachtet der Tatsache, dass die Hersteller der zu bewertenden Medikamente bei ihren Produkten durchweg von einem beträchtlichen oder erheblichen $\mathrm{Zu}$ satznutzen ausgingen. Auch zur Definition der zweckmäßigen Vergleichstherapie gab es unterschiedliche Auffassungen. So hielten die Vertreter der DGHO bei 6 der 10 Prüfmedikamente die jeweiligen Vergleichstherapien für nur bedingt zweckmäßig, da sie zum Zeitpunkt der Studie nicht zweifelsfrei als Therapiestandard angesehen werden konnten. Auch die Wahl der Studienendpunkte und das Vorgehen bei der Erfassung unerwünschter Wirkungen gaben Anlass zu Diskussionen.

Ein gravierendes Problem sieht die DGHO darin, dass die Stimme der Patienten im Bewertungsprozess der Frühen Nutzenbewertung zu wenig Gehör findet. Zwar ist eine Anhörung von Patientenvertretern im Prinzip vorgesehen. Die Patientenorganisationen haben aber nur 3 Wochen Zeit, sich auf Basis des von den Herstellern eingereichten Dossiers in die Materie einzuarbeiten. Das ist für viele Organisationen, deren Arbeit sich auf Ehrenamtliche stützt, zu kurz. Bei mehr als 
Tab. 2. Auszug aus der Verordnung über die Nutzenbewertung von Arzneimitteln nach § 35a Absatz 1 SGB V für Erstattungsvereinbarungen nach $\S 130$ b SGB V

- Ein erheblicher Zusatznutzen liegt vor, wenn eine nachhaltige und gegenüber der zweckmäßigen Vergleichstherapie bisher nicht erreichte große Verbesserung des therapierelevanten Nutzens im Sinne von $\$ 2$ Absatz 3 erreicht wird, insbesondere eine Heilung der Erkrankung, eine erhebliche Verlängerung der Überlebensdauer, eine langfristige Freiheit von schwerwiegenden Symptomen oder die weitgehende Vermeidung schwerwiegender Nebenwirkungen;

- ein beträchtlicher Zusatznutzen liegt vor, wenn eine gegenüber der zweckmäßigen Vergleichstherapie bisher nicht erreichte deutliche Verbesserung des therapierelevanten Nutzens im Sinne von $§ 2$ Absatz 3 erreicht wird, insbesondere eine Abschwächung schwerwiegender Symptome, eine moderate Verlängerung der Lebensdauer, eine für die Patientinnen und Patienten spürbare Linderung der Erkrankung, eine relevante Vermeidung schwerwiegender Nebenwirkungen oder eine bedeutsame Vermeidung anderer Nebenwirkungen;

- ein geringer Zusatznutzen liegt vor, wenn eine gegenüber der zweckmäßigen Vergleichstherapie bisher nicht erreichte moderate und nicht nur geringfügige Verbesserung des therapierelevanten Nutzens im Sinne von $\S 2$ Absatz 3 erreicht wird, insbesondere eine Verringerung von nicht schwerwiegenden Symptomen der Erkrankung oder eine relevante Vermeidung von Nebenwirkungen;

Tab. 3. Nutzenbewertung neuer onkologischer Arzneimittel: abgeschlossene Projekte (Stand: März 2013)

\begin{tabular}{lll}
\hline Indikationsbereich & Substanz & Auftrag \\
\hline Prostatakrebs & Abiraterone (Zytiga) & A11-20 \\
Brustkrebs & Cabazitaxel (Jevtana) & A11-24 \\
Melanom & Ipilimumab (Yervoy) & A12-07 \\
& Vemurafenib (Zelboraf) & A12-08 \\
Schilddrüsenkrebs & Vandetanib (Caprelsa) & A12-09 \\
Nierenzellkrebs & Axitinib (Inlyta) & A12-14 \\
Lungenkrebs & Crizotonib (Xalkori) & A12-15 \\
Non-Hodgkin-Lymphom & Pixantrone (Pixuvri) & A12-17 \\
\hline
\end{tabular}

der Hälfte der IQWiG-Berichte wurde die im Prozess vorgesehene Patientenbeteiligung nicht umgesetzt. Die Konsequenz ist, dass einige der Entscheidungen der letzten 1,5 Jahre einen deutlichen Mangel an Patienteneinbindung erkennen lassen.

\section{Methodische Aspekte bei der Erfassung und Bewertung von Lebensqualitätsparametern in der Onkologie}

\section{Verantwortlicher Autor: Volker Vervölgyi, Köln}

Das zum 1. Januar 2011 in Kraft getretene AMNOG sieht vor, dass sich neue Arzneimittel regelhaft einer Nutzenbewertung unterziehen müssen. Die Nutzenbewertung kann optional auch für bereits im Handel erhältliche Präparate vorgenommen werden. Dafür hat der pharmazeutische Unternehmer ein Dossier vorzulegen, in dem er Belege für den Zusatznutzen im Vergleich zur «Zweckmäßigen Vergleichstherapie» erbringt. Die zweckmäßige Vergleichstherapie wird vom G-BA festgelegt. Die zweckmäßige Vergleichstherapie muss grundsätzlich für die jeweilige Indikation zugelassen sein. Weiterhin muss es sich um den zum Bewertungszeitpunkt verwendeten therapeutischen Standard für die jeweilige Indika- tion handeln. Gibt es mehrere Standards, ist die kostengünstigste Therapie zu wählen (Stand der Information: März 2013).

Die Nutzenbewertung erfolgt in den ersten 3 Monaten nach Marktzulassung eines neuen Arzneimittels. Der G-BA kann das IQWiG oder Dritte mit der Nutzenbewertung beauftragen. In den folgenden 3 Monaten finden beim G-BA ein Stellungnahmeverfahren sowie die Beratung und Entscheidung darüber statt, ob ein gegebenenfalls vom Hersteller behaupteter Zusatznutzen gegenüber der zweckmäßigen Vergleichstherapie anzuerkennen ist.

Für Arzneimittel ohne Zusatznutzen wird die Erstattungshöhe auf den Preis der zweckmäßigen Vergleichstherapie begrenzt, für Arzneimittel mit Zusatznutzen werden die Erstattungsbeträge auf Basis der Nutzenbewertung zwischen dem Hersteller und dem Spitzenverband der Gesetzlichen Krankenversicherungen (GKV) ausgehandelt. Bei der Nutzenbewertung wird geprüft, ob für das neue Arzneimittel ein $\mathrm{Zu}$ satznutzen gegenüber der zweckmäßigen Vergleichstherapie vorliegt, welches Ausmaß und welche Wahrscheinlichkeit der Zusatznutzen für welche Patientengruppen hat.

\section{Zur Begrifflichkeit von «Nutzen» und «Schaden»}

Mit dem Begriff «Nutzen» werden kausal begründete positive Effekte, mit dem Begriff «Schaden» kausal begründete negative Effekte einer medizinischen Intervention auf patientenrelevante Endpunkte bezeichnet [18]. Beide Begriffe beziehen sich auf einen Vergleich mit Placebo (oder einer andersartigen Scheinintervention) oder keiner Behandlung. Wird ein Vergleich mit einer anderen Therapie vorgenommen, wird unter dem Nutzenaspekt von einem «Zusatznutzen» bzw. einem «geringeren Nutzen» gesprochen.

Für Arzneimittel wird bei Vorliegen eines Zusatznutzens bezüglich des Ausmaßes und dessen therapeutischer Bedeutung unter Berücksichtigung des Schweregrades der Erkrankung zwischen «erheblich», «beträchtlich» und «gering» unterschieden (Tab. 2).

Die Nutzenbewertung beruht auf den Ergebnissen wissenschaftlicher Untersuchungen zur Beeinflussung patientenrele- 
Tab. 4. Datenlage zu patientenrelevanten Endpunkten (Stand: März 2013)

\begin{tabular}{|c|c|c|c|c|}
\hline Substanz & $\begin{array}{l}\text { Mortalität } \\
\text { (Gesamtüberleben) }\end{array}$ & Morbidität & Lebensqualität & $\begin{array}{l}\text { Unerwünschte } \\
\text { Ereignisse }\end{array}$ \\
\hline Abiraterone & $\mathrm{X}$ & $\begin{array}{l}\text { X (Schmerz, } \\
\text { Skelettkomplikationen) }\end{array}$ & $(\mathrm{X})$ & $\mathrm{X}$ \\
\hline Cabazitaxel & $\mathrm{X}$ & $\mathrm{X}(\mathrm{PPI})$ & - & $\mathrm{X}$ \\
\hline Eribulin & $\mathrm{X}$ & - & - & $(\mathrm{X})$ \\
\hline Ipilimumab & $\mathrm{X}$ & - & $\mathrm{X}$ & $\mathrm{X}$ \\
\hline Vemurafenib & $\mathrm{X}$ & X (VAS-Schmerz) & $\mathrm{X}$ & $\mathrm{X}$ \\
\hline Vandetanib & \multicolumn{4}{|c|}{ Keine verwertbaren Daten vorhanden } \\
\hline Axitinib & $\mathrm{X}$ & $\mathrm{X}($ FKSI-DRS $)$ & $\mathrm{X}$ & $\mathrm{X}$ \\
\hline Crizotinib & $\mathrm{X}$ & $\begin{array}{l}\text { (X) (QLQ-C30 und QLQ-LC13 } \\
\text { Symptomskalen) }\end{array}$ & $\mathrm{X}$ & $\mathrm{X}$ \\
\hline Pixantrone & \multicolumn{4}{|c|}{ Keine verwertbaren Daten vorhanden } \\
\hline
\end{tabular}

PPI = Present pain intensity, VAS = visuelle Analogskala, FKSI-DRS = Functional Assessment of Cancer Therapy - Kidney Symtom Index - Disease Related Scale, QLQ = Quality of Life Questionnaire, C30 = Core 30, LC13 = Lung Cancer 13. vanter Endpunkte; das sind insbesondere Daten zur Mortalität, Morbidität (Beschwerden und Komplikationen), gesundheitsbezogenen Lebensqualität und zu Nebenwirkungen. Diese Daten werden vom IQWiG regelhaft in die Bewertung einbezogen.

Bisher wurden für 9 onkologische Präparate Nutzenbewertungen durchgeführt (Tab. 3), wobei für 2 der Arzneimittel keine verwertbaren Daten vorlagen. In den anderen Fällen war die Datenlage insgesamt gut, nur in 3 Fällen waren nicht für alle Endpunktkategorien verwertbare Daten verfügbar (Tab. 4).

Für die patientenrelevanten Nutzendimensionen können auch patientenberichtete Endpunkte (PROs) zum Einsatz kommen [19]. Sie geben die subjektiven Eindrücke der Patienten in Bezug auf die Erkrankung wieder und sind somit frei von Interpretationen durch Dritte. Neben der gesundheitsbezogenen Lebensqualität können zum Beispiel auch die Symptome der Erkrankung erfragt werden. Zum Teil werden dafür komplex aufgebaute Fragebögen verwendet. Wie für alle Studienendpunkte so ist auch für PROs zu fordern, dass sie direkt und zuverlässig Änderungen des Gesundheitszustandes abbilden. In den bisher eingereichten Dossiers fehlten teilweise Angaben zur Validierung des gewählten PRO-Erhebungsinstrumentes oder die Auswahl der erfassten Items war nicht nachvollziehbar. Um mithilfe von PROs gewonnene Studienergeb- nisse interpretieren zu können, sollten die verwendeten Messinstrumente den wissenschaftlichen Kriterien hinsichtlich Validität, Reliabilität und Objektivität genügen. Eine weitere Schwierigkeit war, dass die Relevanz der beobachteten Effekte aus den Angaben in den Dossiers nicht abgeschätzt werden konnte. Hier wären aussagekräftige Responderanalysen mit validierten oder anerkannten Responsekriterien wie z.B. einer MID (minimal important difference) notwendig. Liegen solche Responderanalysen nicht vor, muss in der Regel auf den Vergleich der Mittelwerte des Fragebogen-Scores in den Behandlungsgruppen zurückgegriffen werden. Dabei ist die Relevanz des Effektes (mithilfe standardisierter Mittelwertsdifferenzen in Form von Hedges g) zu bewerten [18].

In den meisten klinischen Studien der bereits bewerteten onkologischen Arzneimittel sind Ergebnisse auf Basis von PRO zwar enthalten, sie werden aber vorwiegend als nachgeordnete Zielgröße berichtet. Aus diesem Grund fehlen häufig adäquate Intention-to-Treat-Analysen für diese Endpunkte, wodurch die Ergebnisse eine hohe Anfälligkeit für systematische Verzerrung (Bias) haben.

\section{Disclosure Statement}

Es bestehen keine Interessenkonflikte.

\section{Literatur}

1 Markman M: Progression-free survival versus overall survival as the primary end point in anticancer drug trials: increasingly relevant impact of therapy following progression. Curr Oncol Rep 2009;11:1-

$>2$ Knox J: Progression-free survival as endpoint in metastatic RCC? Lancet 2008;372:427-429.

$\checkmark 3$ Bukowski $\mathrm{R}$ et al.: Effects of sorafenib on symptoms and quality of life: results from a large randomized placebo-controlled study in renal cancer Am J Clin Oncol 2007;3:220-227.
4 Lipscomb J et al.: Patient-reported outcomes in cancer: a review of recent research and policy initiatives. CA Cancer J Clin 2007;57:278-300.

5 Rao D et al.: A comparison of the Renal Cell Carcinoma-Symptom Index (RCC-SI) and the Functional Assessment of Cancer Therapy-Kidney Symptom Index (FKSI). J Pain Symptom Manage 2009;38:291-298

6 Victorson D et al.: Metaanalysis of the correlation between radiographic tumor response and patientreported outcomes. Cancer 2006;106:494-504.
7 Cella D: Quality of life in patients with metastatic renal cell carcinoma: the importance of patient-reported outcomes. Cancer Treat Rev 2009;35:733-737.

8 Cella DF et al.: Reliability and validity of the Functional Assessment of Cancer Therapy-Lung (FACT-L) quality of life instrument. Lung Cancer 1995;12:199-220.

$\checkmark$ Cella D et al.: What is a clinically meaningful change on the Functional Assessment of Cancer TherapyLung (FACT-L) questionnaire? Results from Eastern Cooperative Oncology Group (ECOG) study 5592. J Clin Epidemiol 2002;55:285-295. 
10 Eton DT et al.: Early change in patient-reported health during lung cancer chemotherapy predicts clinical outcomes beyond those predicted by baseline report: results from Eastern Cooperative Oncology Group study 5592. J Clin Oncol 2003;21 1536-1543.

11 Shepherd FA et al.: Erlotinib in previously treated non-small-cell lung cancer. N Engl J Med 2005;353: 123-132.

12 Cella D et al.: Development and validation of the Functional Assessment of Cancer Therapy-Kidney Symptom Index (FKSI). J Support Oncol 2006;4: 191-199.
13 Cella D et al.: Patient-reported outcomes (PROs) in a phase III AXIS trial of axitinib versus sorafenib as second-line therapy for metastatic renal cell carcinoma (mRCC). J Clin Oncol 2011;29(suppl): abstr 4504.

14 Cella D et al.: The Patient-Reported Outcomes Measurement Information System (PROMIS): progress of an NIH Roadmap cooperative group during its first two years. Med Care 2007;45(5 suppl 1):S3-S11.

15 Mukherjee S: The Emperor of All Maladies. A Biography of Cancer. Harper Collins UK, 2011.

16 www.dgho-onkopedia.de/de/onkopedia/leitlinien/ lungenkarzinom-nicht-kleinzellig-nsclc.
17 Eisenhauer EA et al.: New response evaluation criteria in solid tumours: Revised RECIST guideline (version 1,1). Eur J Cancer 2009;45:228-247.

18 Institut für Qualität und Wirtschaftlichkeit im Gesundheitswesen (IQWiG): Allgemeine Methoden, Version 4,0 vom 23,09 2011. Köln, IQWiG, 2011.

19 Patrick D et al.: Patient-reported outcomes; in Higgins JPT, Green S (eds): Cochrane Handbook for Systematic Reviews of Interventions, version 5,0,1. Oxford, The Cochrane Collaboration, 2008. www. cochrane-handbook.org 